FSUJ TPI QO-03/96

March, 1996

\title{
Homodyne detection for measuring internal quantum correlations of optical pulses
}

\author{
T. Opatrnýf, D.-G. Welsch \\ Friedrich-Schiller Universität Jena, Theoretisch-Physikalisches Institut \\ Max-Wien Platz 1, D-07743 Jena, Germany \\ W. Vogel \\ Universität Rostock, Fachbereich Physik \\ Universitätsplatz 3, D-18051 Rostock, Germany
}

\begin{abstract}
A new method is described for determining the quantum correlations at different times in optical pulses by using balanced homodyne detection. The signal pulse and sequences of ultrashort test pulses are superimposed, where for chosen distances between the test pulses their relative phases and intensities are varied from measurement to measurement. The correlation statistics of the signal pulse is obtained from the time-integrated difference photocurrents measured.
\end{abstract}

${ }^{*}$ Permanent address: Palacký University, Faculty of Natural Sciences, Svobody 26, 77146 Olomouc, Czech Republic 
Methods for measuring the quantum statistics of optical fields have been of increasing interest. One of the most fruitful experimental methods has been balanced homodyne detection [1] including homodyne correlation measurements [2]. In particular, combining a single-mode signal field and a strong local oscillator (LO) by a 50\%:50\% beam splitter and measuring the interfering fields in the two output channels by means of two photodetectors, the difference-count statistics yields directly the field-strength statistics of the signal mode for a certain phase parameter. Since knowledge of the probability distributions of the field strengths for all phases within a $\pi$-interval is equivalent to knowledge of the quantum state [3], the field-strength distributions can be used to reconstruct the quantum state in terms of the density matrix or other representative quantities, such as phasespace functions.

In the pioneering experiments by Smithey, Beck, Raymer, and Faridani [4 the Wigner function was determined from the measured field-strength distributions by means of inverse Radon transform and the density-matrix elements in a fieldstrength basis were then obtained as Fourier transforms of the Wigner function (see also Ref. [5]). The method also called optical homodyne tomography (OHT) has been improved in order to obtain the density matrix in a more direct way [6]. In particular, the problem of direct sampling of the density matrix in the photon-number basis has been studied in a number of papers [7]. The method can be extended to the detection of multimode light by increasing the number of the input (and output) channels of the apparatus. The problem of reconstruction of the quantum state of two correlated modes was first considered in Ref. [8], and an analysis of the determination of the quantum state of a correlated $N$-mode field by multiport homodyning is given in Refs. 99.

In the experiments reported in Ref. [4] optical pulses are used and the timeintegrated difference-count statistics is measured. Hence, a single-mode density matrix is reconstructed from the measured data, which only yields information on the quantum statistics of the signal pulse as a whole, but does not contain any information about its internal quantum statistics. Recently, Munroe, Boggavarapu, Anderson, and Raymer [10] have performed OHT experiments using LO pulses that are short compared with the signal pulses. In this way they have been able to obtain the photon-number statistics of a signal pulse at different times in the pulse.

In this paper we propose a method for measuring also the internal correlation statistics of optical pulses, which applies to both classical and nonclassical light. In particular, the method can be used to study correlation-assisted coherence properties in an experiment analogous to Young's interference experiment, but with a "temporal double slit" inside a pulse. Another example is the measurement of the photon-number correlation at different times in the pulse. It is worth noting that even in classical optics the measurement of the internal amplitudephase structure of pulses is not trivial and has been a subject of current interest [11]. 
To measure the internal quantum statistics of an optical pulse, an apparatus is desired that performs an appropriate mode decomposition of the pulse without introduction of additional noise and measures the quantum statistics of the correlated modes. This can be achieved by combining the signal pulse, through a 50\%:50\% beam splitter, with a train of $N$ LO pulses that are short compared with the signal pulse and measuring the time-integrated difference-count statistics in the two output channels of the beam splitter (Fig.1). The signal pulse consists, in general, of a continuum of monochromatic modes with photon destruction and creation operators $\hat{b}(\omega)$ and $\hat{b}^{\dagger}\left(\omega^{\prime}\right)$, respectively, $\left[\hat{b}(\omega), \hat{b}^{\dagger}\left(\omega^{\prime}\right)\right]=\delta\left(\omega^{\prime}-\omega\right)$. Let us suppose that the strong LO pulses are prepared in coherent states, so that the positive-frequency part of the $k$ th pulse centered at time $t_{k}$ can be described by a function $\gamma_{k} g_{k}(t)$, where $g_{k}(t)$ is assumed to be normalized to unity and $\gamma_{k}$ is a complex number. We now consider the operators

$$
\hat{a}_{k}=\int d \omega \tilde{g}_{k}^{*}(\omega) \hat{b}(\omega)
$$

where $\tilde{g}_{k}(\omega)$ is the Fourier transform of $g_{k}(t), \tilde{g}_{k}(\omega) \equiv(2 \pi)^{-1 / 2} \int d \omega g_{k}(t) \exp (i \omega t)$. It can be shown that when the LO pulses $g_{k}(t)$ and $g_{k^{\prime}}(t), k \neq k^{\prime}$, are (approximately) nonoverlapping, then the operators $\hat{a}_{k}$ and $\hat{a}_{k^{\prime}}^{\dagger}$ satisfy the standard bosonic commutation relation $\left[\hat{a}_{k}, \hat{a}_{k^{\prime}}^{\dagger}\right]=\delta_{k k^{\prime}}$. Hence, the train of LO pulses can be used to introduce a set of nonmonochromatic modes [12 and "probe" the correlation statistics of the signal pulse in terms of these modes. In particular, measurement of the integrated difference-count statistics can be shown to be equivalent to measurement of the sum of signal-pulse field strengths

$$
\hat{F}=\sum_{k} q_{k} \hat{F}_{k}\left(\varphi_{k}\right)
$$

Here,

$$
\hat{F}_{k}\left(\varphi_{k}\right)=2^{-1 / 2}\left(\hat{a}_{k} e^{-i \varphi_{k}}+\hat{a}_{k}^{\dagger} e^{i \varphi_{k}}\right)
$$

is the field strength associated with the $k$ th pulse-like nonmonochromatic mode centered at time $t_{k}$ in the signal pulse and $q_{k}$ is a non-negative real parameter describing the relative (square root of the) intensity of the $k$ th LO pulse. Measuring the distribution of the sum field strength (3) for a sufficiently large set of values of $\varphi_{k}$ and $q_{k}$, we may obtain the quantum statistics of the signal pulse within the frame of an $N$-mode density matrix.

The train of LO pulses may be obtained from one generating LO pulse using interferometric methods, so that the distances between the LO pulses, their intensities and relative phases can be controlled. Let us further assume that the signal pulse can be appropriately triggered by the generating LO. In this way, the times $t_{k}$ in the signal pulse and the absolute values and the relative phases of the $F_{k}$ can be controlled. When the "positions" $t_{k}$ of the LO pulses are shifted towards $t_{k}+\Delta t$, then the phases $\varphi_{k}$ are shifted towards $\varphi_{k}+\omega_{0} \Delta t, \omega_{0}$ being the carrier 
frequency of the signal and LO pulses. Since times of the order of magnitude of $\omega_{0}^{-1}$ cannot be controlled, an absolute phase control seems to be impossible when the signal and LO pulses come from different sources. In this case, only quantities averaged with respect to the unknown phase can be obtained.

Let us consider the simplest case when the signal pulse and two short nonoverlapping LO pulses centered at the times $t_{1}$ and $t_{2}$ in the signal pulse are superimposed. In this case, measurement of the time-integrated difference-count statistics yields the statistics of a sum of two-mode field strengths of the signal pulse,

$$
\hat{F}=\hat{F}(\varphi, \Delta \varphi, q)=\hat{F}_{1}(\varphi)+q \hat{F}_{2}(\varphi+\Delta \varphi) .
$$

The measured moments of $\hat{F}$ are related to the moments and correlation functions of the signal pulse at the two times $t_{1}$ and $t_{2}$ in the pulse as

$$
\left\langle\hat{F}^{n}\right\rangle=\sum_{k=0}^{n}\left(\begin{array}{l}
n \\
k
\end{array}\right) q^{k}\left\langle\hat{F}_{1}^{n-k}(\varphi) \hat{F}_{2}^{k}(\varphi+\Delta \varphi)\right\rangle .
$$

Measuring $\left\langle\hat{F}^{n}\right\rangle$ for $n+1$ values of $q$, from Eq. (5) one obtains a set of $n+1$ linear algebraic equations whose solution yields the signal-pulse moments and correlation functions $\left\langle\hat{F}_{1}^{n-k}(\varphi) \hat{F}_{2}^{k}(\varphi+\Delta \varphi)\right\rangle$ for the chosen values of $\varphi$ and $\Delta \varphi$. Varying $\varphi$ and $\Delta \varphi$, the procedure can be repeated many times to obtain the $\varphi$ and $\Delta \varphi$ dependences of the moments and correlation functions. In the limit when the number of measurements goes to infinity all the moments and correlations can be obtained, the knowledge of which is equivalent to knowledge of the twomode density matrix. Clearly, when the phase $\varphi$ cannot be controlled the phaseaveraged (even) moments

$$
\overline{\left\langle\hat{F}^{n}\right\rangle} \equiv \frac{1}{2 \pi} \int d \varphi\left\langle\hat{F}^{n}\right\rangle
$$

can only be measured and Eq.(5) modifies to

$$
\overline{\left\langle\hat{F}^{n}\right\rangle}=\sum_{k=0}^{n}\left(\begin{array}{l}
n \\
k
\end{array}\right) q^{k} \overline{\left\langle\hat{F}_{1}^{n-k}(\varphi) \hat{F}_{2}^{k}(\varphi+\Delta \varphi)\right\rangle}
$$

$n$ being even.

Let us briefly discuss the situation of nonperfect detection. In this case, $\hat{a}_{k}$ must be replaced by $\eta \hat{a}_{k}+\sqrt{\eta(1-\eta)} \hat{c}_{k}$ (cf. Ref. [13]), where $\eta$ is the detection efficiency $(\eta \leq 1)$ and $\hat{c}_{k}$ and $\hat{c}_{k}^{\dagger}$ are bosonic noise operators. The corresponding noise modes can be considered to be in vacuum states, so that for each of these modes the $m$ th moment of the corresponding field strength is given by $2^{-m / 2}(m-$ $1) ! !$ for even $m$ and zero for odd $m[(m-1) ! !=1 \cdot 3 \cdots(m-3)(m-1)$ for $m \geq 2$; $(-1) ! ! \equiv 1]$. We then find that in the sum in Eq. (đ) the replacement

$$
\begin{aligned}
\overline{\left\langle\hat{F}_{1}^{n-k}(\varphi) \hat{F}_{2}^{k}(\varphi+\Delta \varphi)\right\rangle} & \rightarrow \sum_{l, m}\left(\begin{array}{c}
n-k \\
l
\end{array}\right)\left(\begin{array}{c}
k \\
m
\end{array}\right) \eta^{\frac{n+l+m}{2}}\left(\frac{1-\eta}{2}\right)^{\frac{n-l-m}{2}} \\
\times(n-k-l-1) ! !(k-m-1) ! ! & \left\langle\hat{F}_{1}^{l}(\varphi) \hat{F}_{2}^{m}(\varphi+\Delta \varphi)\right\rangle
\end{aligned}
$$


must be made. When $k$ is even (odd) the $l$ - and $m$-sums in Eq. (8) run over the even (odd) integers between zero and $n-k$ and between zero and $k$, respectively. The replacement means that the reconstructed moments and correlations in Eq. (7) are attenuated and "contaminated" by the moments of lower orders. Nevertheless, we can recurrently correct them by subtracting the contaminating terms, because only lower-order terms are included.

To illustrate the method, let us briefly consider the phase-averaged secondand forth-order moments of the measured time-integrated difference-count statistics. Information about the lowest-order correlation in the signal pulse at two times $t_{1}$ and $t_{2}$ is obtained from the second-order moment,

$$
\overline{\left\langle\hat{F}^{2}\right\rangle}=\overline{\left\langle\hat{F}_{1}^{2}(\varphi)\right\rangle}+q^{2} \overline{\left\langle\hat{F}_{2}^{2}(\varphi)\right\rangle}+2 q \overline{\left\langle\hat{F}_{1}(\varphi) \hat{F}_{2}(\varphi+\Delta \varphi)\right\rangle} .
$$

Whereas $\overline{\left\langle\hat{F}_{1}^{2}(\varphi)\right\rangle}$ and $\overline{\left\langle\hat{F}_{2}^{2}(\varphi)\right\rangle}$ are closely related to the mean numbers of photons at the two times in the signal pulse

$$
\overline{\left\langle\hat{F}_{k}^{2}(\varphi)\right\rangle}=\left\langle\hat{n}_{k}\right\rangle+\frac{1}{2}
$$

$(k=1,2)$, the cross term

$$
\overline{\left\langle\hat{F}_{1}(\varphi) \hat{F}_{2}(\varphi+\Delta \varphi)\right\rangle}=\frac{1}{2}\left\langle\hat{a}_{1}^{\dagger} \hat{a}_{2}\right\rangle e^{-i \Delta \varphi}+\text { c.c. }
$$

as a function of the difference phase $\Delta \varphi$ can be regarded as the second-order coherence function that probes the effect of signal-pulse interference at a "temporal double slit" in the pulse [14. Choosing $q=1$ and measuring the second-order moment $\overline{\left\langle\hat{F}^{2}\right\rangle}$ for a set of phase differences $\Delta \varphi$ and $\Delta \varphi+\pi$, the interference term is simply given by the difference of the measured moments at the two phase differences. In the case of nonperfect detection the photon-number terms are attenuated and contaminated by a constant,

$$
\overline{\left\langle\hat{F}_{k}^{2}(\varphi)\right\rangle} \rightarrow \eta^{2} \overline{\left\langle\hat{F}_{k}^{2}(\varphi)\right\rangle}+\frac{1}{2} \eta(1-\eta),
$$

whereas the interference term is only attenuated,

$$
\overline{\left\langle\hat{F}_{1}(\varphi) \hat{F}_{2}(\varphi+\Delta \varphi)\right\rangle} \rightarrow \eta^{2} \overline{\left\langle\hat{F}_{1}(\varphi) \hat{F}_{2}(\varphi+\Delta \varphi)\right\rangle} .
$$

Extending the measurement to the fourth-order moments $\overline{\left\langle\hat{F}^{4}\right\rangle}$ for different values of $q$ and $\Delta \varphi$, the phase-averaged fourth-order moments and and correlations of $\hat{F}_{1}(\varphi)$ and $\hat{F}_{2}(\varphi)$ can be obtained. The moments $\overline{\left\langle\hat{F}_{1}^{4}(\varphi)\right\rangle}$ and $\overline{\left\langle\hat{F}_{2}^{4}(\varphi)\right\rangle}$ carry information about the photon-number statistics (first- and second-order moments of the photon numbers) of the signal pulse at two different times in the pulse. The two-time correlations are given by

$$
\overline{\left\langle\hat{F}_{1}^{3}(\varphi) \hat{F}_{2}(\varphi+\Delta \varphi)\right\rangle}=\frac{3}{2} \overline{\left\langle\hat{F}_{1}(\varphi) \hat{F}_{2}(\varphi+\Delta \varphi)\right\rangle}+\frac{3}{4}\left(\left\langle\hat{a}_{1}^{\dagger 2} \hat{a}_{1} \hat{a}_{2}\right\rangle e^{-i \Delta \varphi}+\text { c.c. }\right),
$$




$$
\overline{\left\langle\hat{F}_{1}(\varphi) \hat{F}_{2}^{3}(\varphi+\Delta \varphi)\right\rangle}=\frac{3}{2} \overline{\left\langle\hat{F}_{1}(\varphi) \hat{F}_{2}(\varphi+\Delta \varphi)\right\rangle}+\frac{3}{4}\left(\left\langle\hat{a}_{2}^{\dagger 2} \hat{a}_{2} \hat{a}_{1}\right\rangle e^{i \Delta \varphi}+\text { c.c. }\right)
$$

with $\overline{\left\langle\hat{F}_{1}(\varphi) \hat{F}_{2}(\varphi+\Delta \varphi)\right\rangle}$ from Eq. (11), and

$$
\overline{\left\langle\hat{F}_{1}^{2}(\varphi) \hat{F}_{2}^{2}(\varphi+\Delta \varphi)\right\rangle}=\frac{1}{4}\left(\left\langle\hat{a}_{1}^{\dagger 2} \hat{a}_{2}^{2}\right\rangle e^{-i 2 \Delta \varphi}+\text { c.c. }\right)+\left\langle\left(\hat{n}_{1}+\frac{1}{2}\right)\left(\hat{n}_{2}+\frac{1}{2}\right)\right\rangle \text {. }
$$

In particular, from Eq. (16) together with the mean photon numbers obtained from the second-order moments, Eq. (10), the photon-number correlation at the times $t_{1}$ and $t_{2}$ in the signal pulse can be determined, which for stationary ergodic fields is usually measured in the Hanbury-Brown and Twiss experiment. When the detection efficiency is less than unity the terms $\overline{\left\langle\hat{F}_{k}^{4}(\varphi)\right\rangle}$ changes as

$$
\overline{\left\langle\hat{F}_{k}^{4}(\varphi)\right\rangle} \rightarrow \eta^{4} \overline{\left\langle\hat{F}_{k}^{4}(\varphi)\right\rangle}+3 \eta^{3}(1-\eta) \overline{\left\langle\hat{F}_{k}^{2}(\varphi)\right\rangle}+\frac{3}{4} \eta^{2}(1-\eta)^{2}
$$

and with respect to the symmetrical cross term (16) we find that

$$
\begin{aligned}
& \overline{\left\langle\hat{F}_{1}^{2}(\varphi) \hat{F}_{2}^{2}(\varphi+\Delta \varphi)\right\rangle} \rightarrow \eta^{4} \overline{\left\langle\hat{F}_{1}^{2}(\varphi) \hat{F}_{2}^{2}(\varphi+\Delta \varphi)\right\rangle} \\
& +\frac{1}{2} \eta^{3}(1-\eta)\left[\overline{\left\langle\hat{F}_{1}^{2}(\varphi)\right\rangle}+\overline{\left\langle\hat{F}_{2}^{2}(\varphi)\right\rangle}\right]+\frac{1}{4} \eta^{2}(1-\eta)^{2} .
\end{aligned}
$$

The inclusion in the analysis of higher-order moments is straightforward. In summary, the proposed method may serve as a useful tool for determining the correlation statistics of optical pulses, with special emphasis on nonclassical light. The application to pulses becomes feasible since only ensemble averaging is used. The other advantage of the method is that it also applies to weak quantum light, because homodyning (with strong LO) "amplifies" the signal. In particular, the method can be used to obtain the correlation statistics of pulses of the type used in Ref. [10] for studying the photon-number statistics at different times in a pulse. In the paper we have restricted attention to the determination of moments and correlations at different times in the signal pulse. The problem of reconstruction of the associated density matrices will be considered in a forthcoming paper.

This work was supported by the Deutsche Forschungsgemeinschaft. One of us (T.O.) is grateful to J. Peřina for discussion and acknowledges a support of an internal grant of the Palacký University. 


\section{References}

[1] H. P. Yuen and J.H. Shapiro, IEEE Trans. Inform. Theor. 26, 78 (1980); H. P. Yuen and V.W.S. Chan, Opt. Lett. 8, 177 (1983); N. G. Walker, J. Mod. Opt. 34, 15 (1987); S. Braunstein, Phys. Rev. A42, 474 (1990); W. Vogel and J. Grabow, Phys. Rev. A47, 4227 (1993).

[2] Z.Y. Ou, C.K. Hong, and L. Mandel, Phys. Rev. A36, 192 (1987); W. Vogel, Phys. Rev. Lett. 67, 2450 (1991); Phys. Rev. A51, 4160 (1995).

[3] K. Vogel and H. Risken, Phys. Rev. A40, 2847 (1989).

[4] D.T. Smithey, M. Beck, M.G. Raymer, and A. Faridani, Phys. Rev. Lett. 70, 1244 (1993).

[5] D.T. Smithey, M. Beck, J. Cooper, M. G. Raymer, and A. Faridani, Phys. Scr. T48, 35 (1993).

[6] H. Kühn, D.-G. Welsch, and W. Vogel, J. Mod. Opt. 41, 1607 (1994); W. Vogel and D.-G. Welsch, Lectures on Quantum Optics (Akademie-Verlag, Berlin, 1994); G.M. D’Ariano, C. Machiavello, and M.B.A. Paris, Phys. Rev. A50, 4298 (1994); Phys. Lett. 195A, 31 (1994).

[7] G.M. D'Ariano, Quantum Semiclass. Opt. 7, 693 (1995); G.M. D'Ariano, U. Leonhardt, and H. Paul, Phys. Rev. A52, R1881 (1995); U. Leonhardt, H. Paul, and G.M. D'Ariano, Phys. Rev. A52, 4899 (1995); H. Paul, U. Leonhardt, and G.M. D'Ariano, Acta Phys. Slov. 45, 261 (1995); Th. Richter, Phys. Lett. A, in press; U. Leonhardt, M. Munroe, T. Kiss, M.G. Raymer, and Th. Richter, Opt. Commun., in press.

[8] M.G. Raymer, D.T. Smithey, M.Beck, M. Anderson, and D.F. McAlister, Third International Wigner Symposium (Oxford, 1993).

[9] H. Kühn, D.-G. Welsch, and W. Vogel, Phys. Rev. A51, 4240 (1995); W. Vogel and D.-G. Welsch, Acta Phys. Slov. 45, 313 (1995); A. Zucchetti, W. Vogel, and D.-G. Welsch, Phys. Rev. A, in press.

[10] M. Munroe, D. Boggvarapu, M.E. Anderson, and M.G. Raymer, Phys. Rev. A52, R924 (1995).

[11] J.-C. Diels, J.J. Fontaine, I.C. McMichael, and F. Simoni, Appl. Opt. 24, 1270 (1985); K. Naganuma, K. Mogi, and H. Yamada, IEEE J. Quantum Electron. 25, 1225 (1989); Appl. Phys. Lett. 54, 1201 (1989); J.L.A. Chilla and O.E. Martinez, IEEE J. Quantum Electron. 27, 1228 (1991); C. Yan and J.C. Diels, J. Opt. Soc. Am. B8, 1259 (1991); D.J. Kane and R. Trebino, Opt. Lett. 18, 823 (1993); M. Beck, M.G. Raymer, I.A. Walmsley, and V. 
Wong, Opt. Lett. 18, 2041 (1993); K.W. DeLong, R. Trebino, and D.J. Kane, J. Opt. Soc. Am. B11, 1595 (1994).

[12] U.M. Titulaer and R.J. Glauber, Phys. Rev. 145, 1041 (1966).

[13] B. Yurke and D. Stoler, Phys. Rev. A 36, 1955 (1987).

[14] For ordinary second-order coherence see, e.g., J. Peřina, Coherence of Light (2nd. ed., Reidel, Dordrecht, 1985).

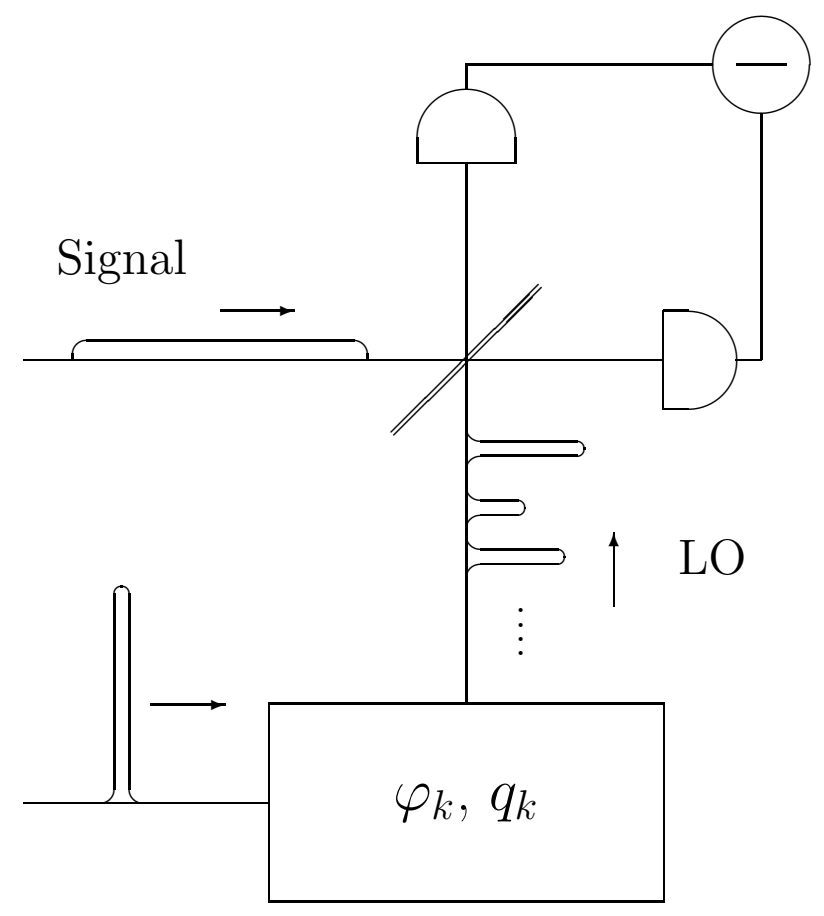

Figure 1: Scheme of the measurement. The signal pulse and a train of strong local-oscillator (LO) pulses that are short compared with the signal pulse are combined by a $50 \%: 50 \%$ beam splitter, and the time-integrated difference-count statistics in the output channels is measured. The train of LO pulses is produced interferometrically, so that the pulse distances, relative phases, and intensities can be controlled. 